\title{
The effects of antimony doping on the surface structure of rutile $\mathrm{TiO}_{2}(110)$
}

\author{
Ralf Bechstein $^{1}$, Mitsunori Kitta ${ }^{2,3}$, Jens Schütte ${ }^{1}$, Hiroshi Onishi ${ }^{2}$ \\ and Angelika Kühnle ${ }^{1}$ \\ ${ }^{1}$ Fachbereich Physik, Universität Osnabrück, Barbarastraße 7, D-49076 Osnabrück, Germany \\ ${ }^{2}$ Department of Chemistry, Kobe University, Rokko-dai, Nada-ku, Kobe 657-8501, Japan \\ E-mail: kuehnle@uos.de
}

Received 2 February 2009, in final form 11 March 2009

Published 10 June 2009

Online at stacks.iop.org/Nano/20/264003

\begin{abstract}
Titanium dioxide represents a very important wide bandgap photocatalyst that is known to be sensitized to visible light by transition metal doping. Antimony doping has been demonstrated to provide photocatalytic activity when codoped with chromium at an optimum dopant ratio $[\mathrm{Sb}] /[\mathrm{Cr}]$ of about 1.5 . Here, the role of antimony doping on the surface structure of rutile $\mathrm{TiO}_{2}(110)$ is studied using non-contact atomic force microscopy (NC-AFM) under ultra-high vacuum conditions. At first glance, the surface structure of antimony-doped $\mathrm{TiO}_{2}(110)$ resembles the structure of pristine $\mathrm{TiO}_{2}(110)$. However, in contrast to what is found in pristine $\mathrm{TiO}_{2}(110)$, a dense layer of protruding features is observed upon antimony doping, which is tentatively ascribed to antimony-rich clusters. Moreover, homogeneously distributed holes are found on the surface, which differ in depth and shape depending on the preparation conditions. Holes with depths ranging from a few up to more than a hundred monatomic steps are observed. These holes are explained by surface segregation of antimony during annealing, as the ionic radius of $\mathrm{Sb}^{3+}$ is considerably larger than the ionic radius of $\mathrm{Ti}^{4+}$. Our finding provides an indication of why an antimony concentration larger than the optimum ratio results in decreased photocatalytic activity. Moreover, controlling annealing temperature seems to constitute a promising strategy for creating nanosized holes on $\mathrm{TiO}_{2}$ surfaces.
\end{abstract}

(Some figures in this article are in colour only in the electronic version)

\section{Introduction}

Titanium dioxide constitutes a very prominent and widely used example of a wide bandgap photocatalyst $[1,2]$, which is of high importance for the degradation of toxic compounds [3] and for the production of hydrogen [4]. It has been demonstrated that $\mathrm{TiO}_{2}$ can be successfully sensitized to visible light by doping with foreign elements, providing an additional donor or acceptor level in the bandgap of $\mathrm{TiO}_{2}$ [5]. In particular, doping with transition metals has been reported to result in an intense absorption of visible light $[6,7]$. Among those transition metals, chromium has also been studied, revealing high visible-light absorption in reflection spectroscopy measurements [8]. However, despite the absorption in the visible-light region, chromium-doped $\mathrm{TiO}_{2}$ has been found to be photocatalytically inactive [8]. This has

3 Present address: Department of Chemistry, School of Science, Osaka University, Mihogaoka, Ibaraki, Osaka 567-0047, Japan. been explained by the creation of additional oxygen vacancies upon chromium doping [8], as vacancies might constitute recombination sites for electron-hole pairs. Recent noncontact atomic force microscopy (NC-AFM) measurements have provided experimental evidence for vacancy creation upon chromium doping, further supporting this picture [9].

Interestingly, upon codoping with chromium and antimony, rutile titania particles have shown both visible-light absorption as well as photocatalytic activity. The rate of the photocatalytic $\mathrm{O}_{2}$ production has been found to be optimized at a ratio of about 1.5, i.e. in excess of antimony [10, 8]. Even larger antimony excess has been observed to again result in reduced photocatalytic activity. The excess antimony most likely exists in equal amounts of antimony ions with the oxidation states $\mathrm{Sb}^{3+}$ and $\mathrm{Sb}^{5+}$, replacing $\mathrm{Ti}^{4+}$ in the lattice. Consequently, they auto-compensate for charging. NC-AFM images of chromium- and antimony-codoped $\mathrm{TiO}_{2}$ have revealed two important aspects, namely the absence of additional oxygen 
vacancies in the case of codoping and a firm integration of the dopant atoms into the crystal lattice [11].

Here, we address the role of excess antimony in chromium- and antimony-codoped titania. The existence of antimony trivalent cations $\mathrm{Sb}^{3+}$ with a comparatively large ionic radius of $76.0 \mathrm{pm}$ compared to $60.0 \mathrm{pm}$ for $\mathrm{Sb}^{5+}$ and $60.5 \mathrm{pm}$ for the replaced titanium ions $\mathrm{Ti}^{4+}$ [12] has been suggested to result in a deformed $\mathrm{TiO}_{2}$ lattice [8]. In the present work, we study the influence of antimony on the surface structure of rutile $\mathrm{TiO}_{2}(110)$ using high-resolution NC-AFM. Our results reveal that antimony-doped $\mathrm{TiO}_{2}$, at a first glance, resembles the structure of pristine $\mathrm{TiO}_{2}(110)$. However, a dense layer of protruding features is observed, which is ascribed to antimony-rich clusters. Besides these clusters, different types of holes are observed on the surface. Shape and depth of these holes appear to depend on the annealing parameters. A possible scenario is given, explaining the formation of these holes based on the large ionic radius of $\mathrm{Sb}^{3+}$.

\section{Experimental section}

The scanning probe technique used in this study is NCAFM [13] operated in the frequency modulation mode [14]. For this technique, a sharp nanoscopic tip mounted at the end of an oscillating cantilever is scanned in close proximity over the sample surface while keeping the oscillation amplitude constant. The forces acting between tip and surface atoms shift the cantilever's eigenfrequency. This frequency shift can be directly used as an imaging signal when scanning the cantilever at a fixed tip-surface distance (constant height mode). Alternatively, the frequency shift can be kept constant to measure the height profile of equal frequency shift (referred to as topography). Measurements are performed at room temperature in an ultra-high vacuum (UHV) chamber (base pressure below $10^{-10}$ mbar) equipped with a VT AFM 25 (Omicron, Taunusstein, Germany) and an easy-PLL Plus phase-locked loop controller (Nanosurf, Liestal, Switzerland) for oscillation excitation and signal demodulation. We use silicon cantilevers (type PPP-NCH) from NanoWorld (Neuchâtel, Switzerland) with an eigenfrequency of about $300 \mathrm{kHz}$ operated at an amplitude of $10 \mathrm{~nm}$. For these experiments, the cantilevers are sputtered with $\mathrm{Ar}^{+}$ions after insertion into the chamber. The $\mathrm{TiO}_{2}$ samples are crystals of the highest quality available (MTI, Richmond, MD, USA). The crystals were doped with antimony by calcining them together with $\mathrm{Sb}_{2} \mathrm{O}_{3}$ powder in a crucible at $1420 \mathrm{~K}$ for $10 \mathrm{~h}$ in air [8]. The total amount of antimony in the doped crystal is difficult to quantify. However, in the upper few micrometers the antimony concentration is known to be of the order of 3 at.\% [10]. This order of magnitude was confirmed by x-ray photoelectron spectroscopy (XPS) measurements of the freshly calcined wafer. After insertion into the UHV chamber, the surface was cleaned by cycles of $15 \mathrm{~min}^{+}$ion sputtering at $1 \mathrm{keV}$ and subsequent annealing. The sample was either annealed for $15 \mathrm{~min}$ at $1100 \mathrm{~K}$ or for $1 \mathrm{~h}$ at $930 \mathrm{~K}$. The preparation parameters were chosen in accordance with our experience on pristine $\mathrm{TiO}_{2}(110)$. In order to minimize longrange electrostatic interactions, a bias voltage in the range of $-0.6 \mathrm{~V}$ to $-1.4 \mathrm{~V}$ was applied to the tip.
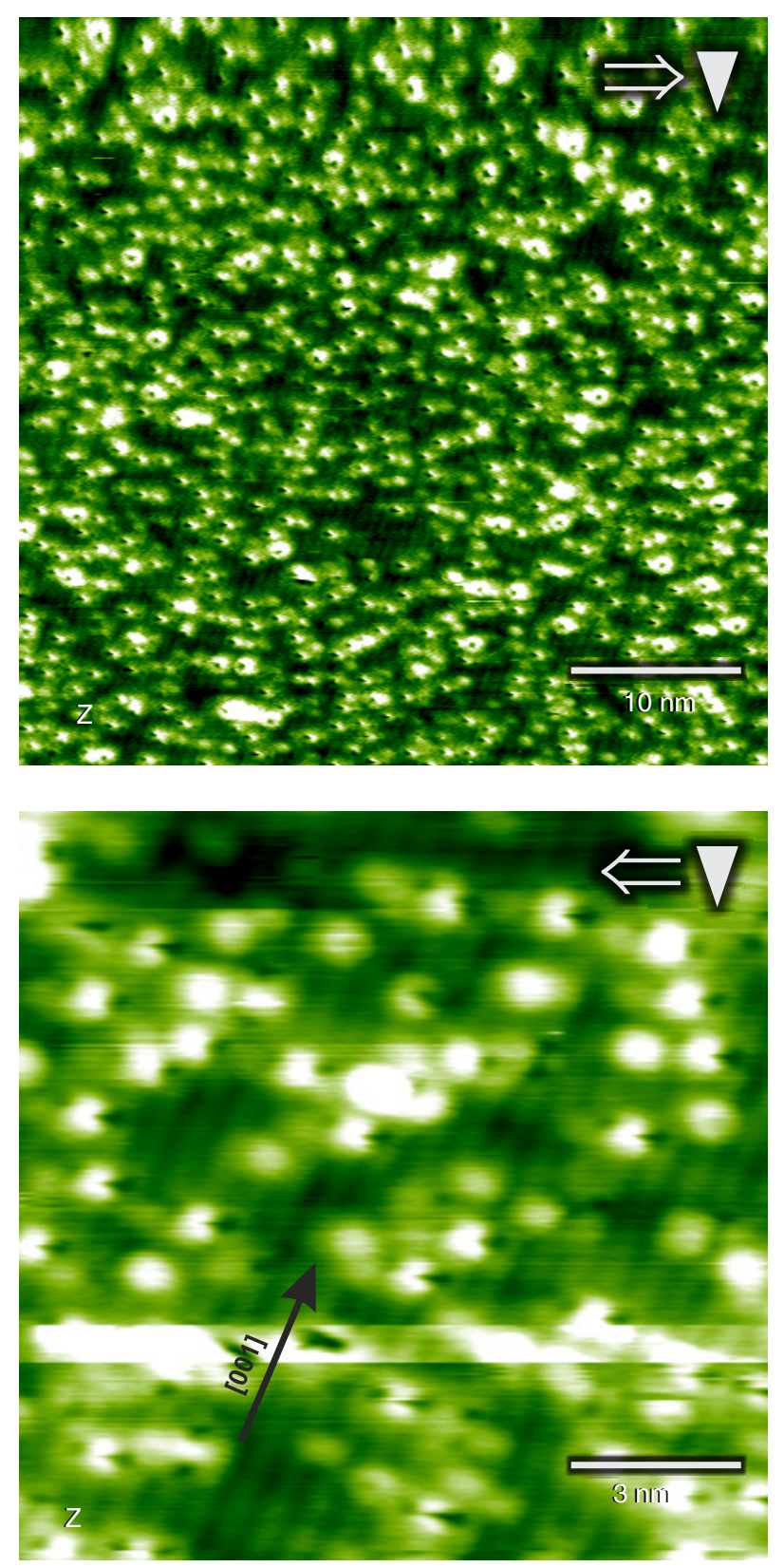

Figure 1. NC-AFM topography images obtained on antimony-doped $\mathrm{TiO}_{2}(110)$ after annealing at $1100 \mathrm{~K}$. The surface is found to be covered by a dense layer of protruding features, which is tentatively ascribed to antimony-rich clusters. The dark spot in the center of each cluster is a scan artifact, which can be explained by slow distance feedback loop settings as described in [17]. Besides the clusters, a row pattern resembling the $\mathrm{TiO}_{2}(110)$ surface can be identified in both images. The periodicity perpendicular to [001] was measured to be $(660 \pm 30) \mathrm{pm}$.

\section{Results and discussion}

Here, the investigation of a $\mathrm{TiO}_{2}(110)$ sample doped with an antimony concentration of about 3 at.\% is presented. We first describe the surface structure at the atomic scale and compare it to the surface structure of pristine titania. Second, the surface structure at larger frame size is presented, which is dominated by the formation of holes of different shape and depth. In the third part we highlight the influence of the annealing procedure on the hole formation. 


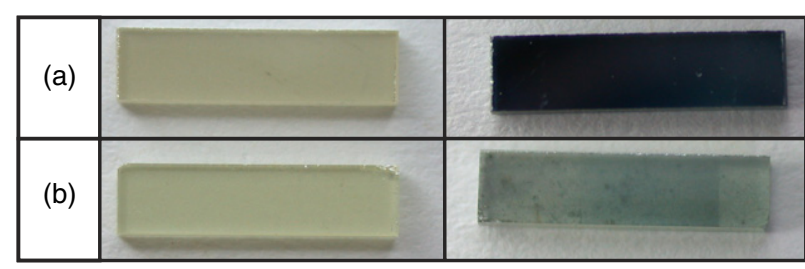

Figure 2. Photographs of the pristine (a) and the antimony-doped (b) $\mathrm{TiO}_{2}(110)$ sample before (left) and after (right) preparation in UHV. The pristine sample was treated by about 30 cycles of sputtering and annealing. The doped sample was treated by 8 preparation cycles. All samples are of dimensions $8 \times 2 \times 1 \mathrm{~mm}^{3}$.

\subsection{Structure at the atomic scale}

The surface structure of antimony-doped titania at the atomic scale is shown in figure 1. The surface is found to be covered by a dense layer of 5-6\% of a monolayer of protruding features (1 ML corresponds to one feature per unit cell). The apparent height of these features was measured to be of the order of $100 \mathrm{pm}$. This is very similar to what has been measured as the apparent height of hydroxyl defects on $\mathrm{TiO}_{2}(110)$ [15]. However, the features observed here appear to have a much larger diameter than typical hydroxyl defects, indicating that the features observed here might be of a different nature. Moreover, if the observed features would be hydroxyl defects, the sample would be heavily reduced. Such a reduced sample is tied to a dark blue color of the entire crystal. In contrast, the crystal is found to be transparent after calcination and only slightly bluish after UHV treatment, as shown in figure 2(b). We cannot rule out that antimony doping results in surface rather than bulk reduction due to external strain [16], which would not necessarily be accompanied by a dark blue color. However, as outlined below, we favor the assignment of the protruding features being antimony-rich clusters rather than hydroxyl defects.

Besides the clusters, the typical row pattern of the $\mathrm{TiO}_{2}$ (110) surface is also visible in the images in figure 1. The periodicity perpendicular to the [001] direction was measured to be $(660 \pm 30) \mathrm{pm}$, which is within the error identical to the surface unit cell length of the pristine titania surface.

Figure 3 reveals that the clusters cover all terraces and islands of the surface. Interestingly, the clusters seem not to adsorb in close proximity to the step edges. In a thin stripe at the edges of the islands (indicated by red arrow), the line pattern of the surface is visible. From a Fourier transformation of this image (not shown), the lines are measured to be separated by $(670 \pm 30) \mathrm{pm}$, similar to what was obtained from figure 1. The findings at the atomic scale are independent of the applied annealing recipes (i.e. $15 \mathrm{~min}$ at $1100 \mathrm{~K}$ or $1 \mathrm{~h}$ at $930 \mathrm{~K})$. In particular, the density of clusters was found to be virtually identical after each preparation cycle.

\subsection{Holes}

A typical image of large frame size obtained on the antimonydoped titania surface is presented in figure 4. The terraces are found to be formed similar to what we typically observe on pristine $\mathrm{TiO}_{2}(110)$. The monatomic step height was measured to be identical to what we found on pristine titania.
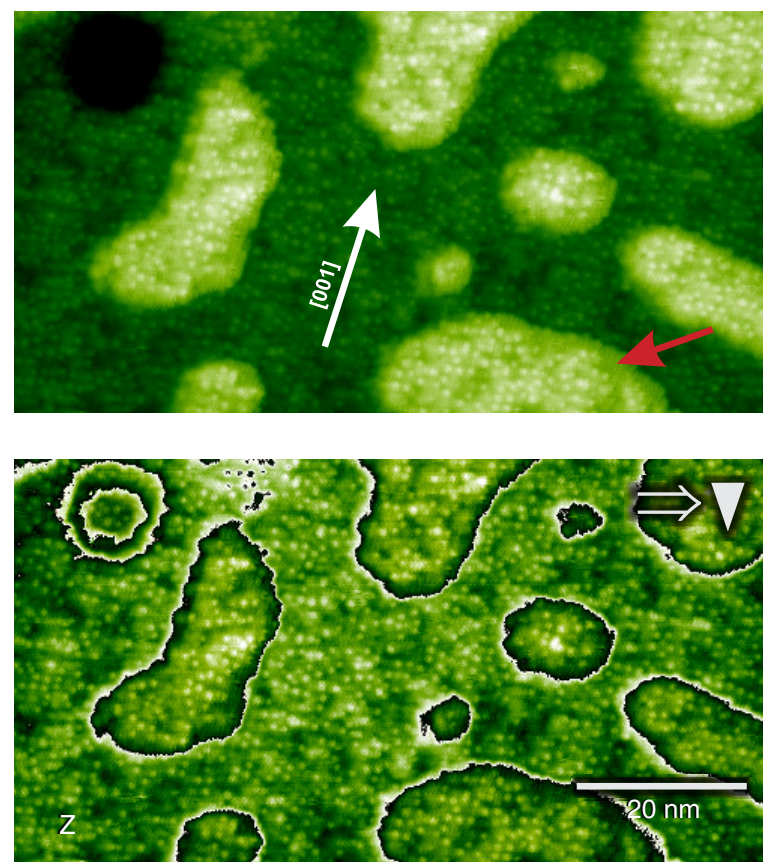

Figure 3. NC-AFM topography image obtained on antimony-doped $\mathrm{TiO}_{2}(110)$ annealed at $930 \mathrm{~K}$. The identical image is presented in normal color scale (upper image) and in repeated color scale (lower image). The clusters cover all terraces. However, the regions on the islands near the step edges are found to be free from clusters, as indicated by the red arrow. There, the typical row pattern known from the pristine surface is visible.

Furthermore, two deep holes are visible in figure 4(a) with a diameter of about $50 \mathrm{~nm}$. The depth of these holes cannot be obtained from NC-AFM measurements as explained in the following. The line profiles presented in figure 4 show a horizontal scan line in the forward direction of the topography (c), the detuning (d) and the amplitude (e) image. At the beginning of the hole the detuning drops to zero, indicating a too large tip-sample distance. The topography signal shows that the scanner approaches the sample at the hole site by more than $10 \mathrm{~nm}$, but the detuning remains zero. Apparently, the base of the hole was not reached by the tip. At the end of the hole, the tip strongly interacts with the backside of the hole, which is indicated by large negative and even positive detuning values. This coincides with a decrease in amplitude of about $10 \%$, indicating a highly dissipative interaction. This behavior was observed at all hole sites, even with the fastest distance feedback loop settings available. From various attempts to scan such holes at small frame size (not shown), the typical depth can be assumed to be definitely larger than $30 \mathrm{~nm}$. Assuming an aspect ratio of the tip of typically 2 at the highest, which corresponds to a cone angle of the tip apex of at least $90^{\circ}$, the depth of a $50 \mathrm{~nm}$ wide hole is - as a matter of principle-not accessible if the depth is larger than $25 \mathrm{~nm}$.

The holes are found to be rather homogeneously distributed at the surface as indicated by figure 5 . The mean distance between neighboring holes was measured to be about $1 \mu \mathrm{m}$. Interestingly, all holes are found to be surrounded by small islands as depicted by the detailed images in figure 5, resembling a volcano-like crater. 

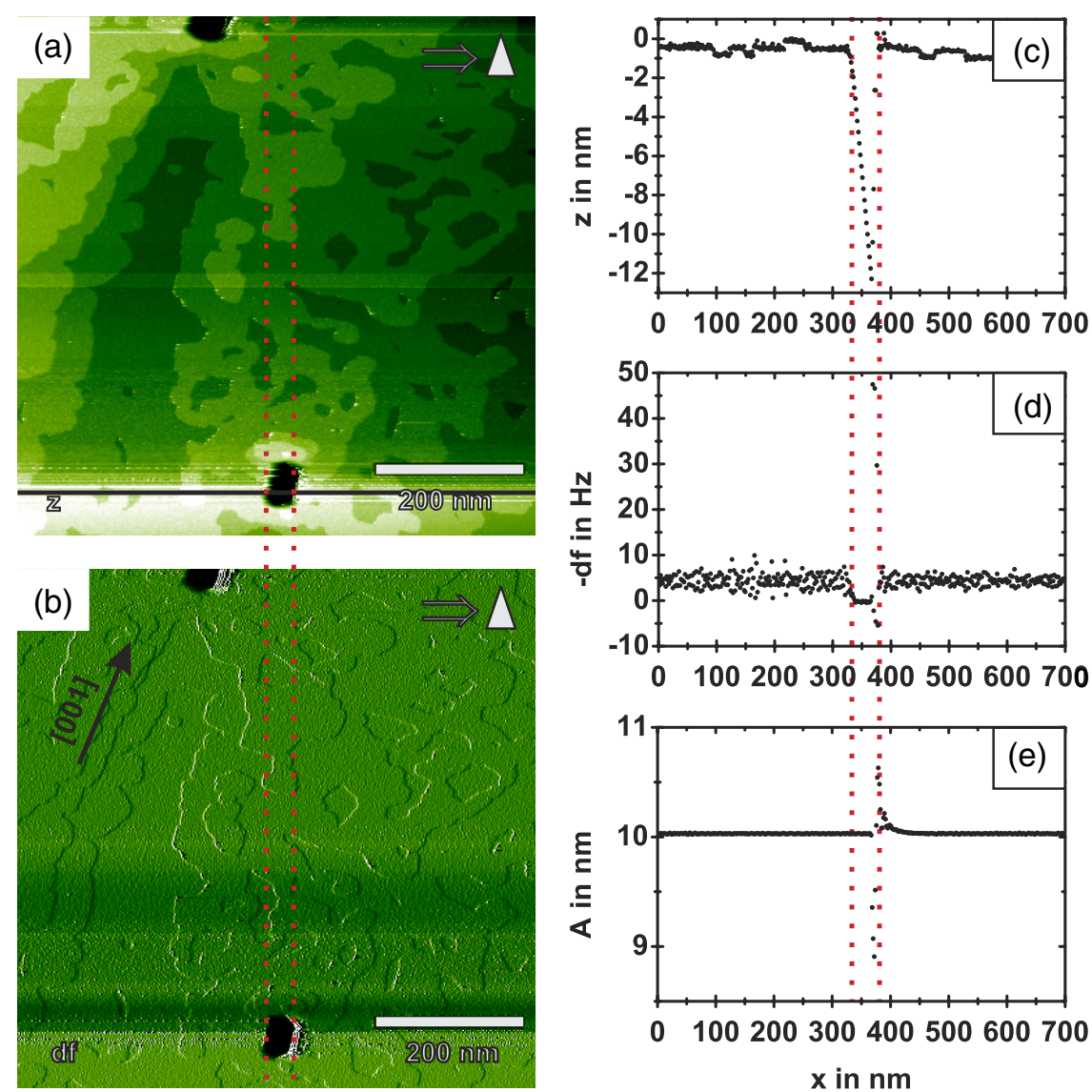

Figure 4. NC-AFM topography (a) and detuning (b) image obtained on antimony-doped $\mathrm{TiO}_{2}(110)$. Two holes can be identified with a diameter of about $50 \mathrm{~nm}$. Line profiles depict a horizontal scan line in forward direction of the topography (c), the detuning (d) and the amplitude (e) signal at a hole site (indicated by the black line). The line profiles indicate that the hole must be deeper than $12 \mathrm{~nm}$ as the detuning remains zero within the hole while the scanner approaches the surface. At the backside of the hole, the tip strongly interacts with the sample as the scanner cannot retract the tip fast enough. This is indicated by even positive detuning values and a decrease in amplitude.
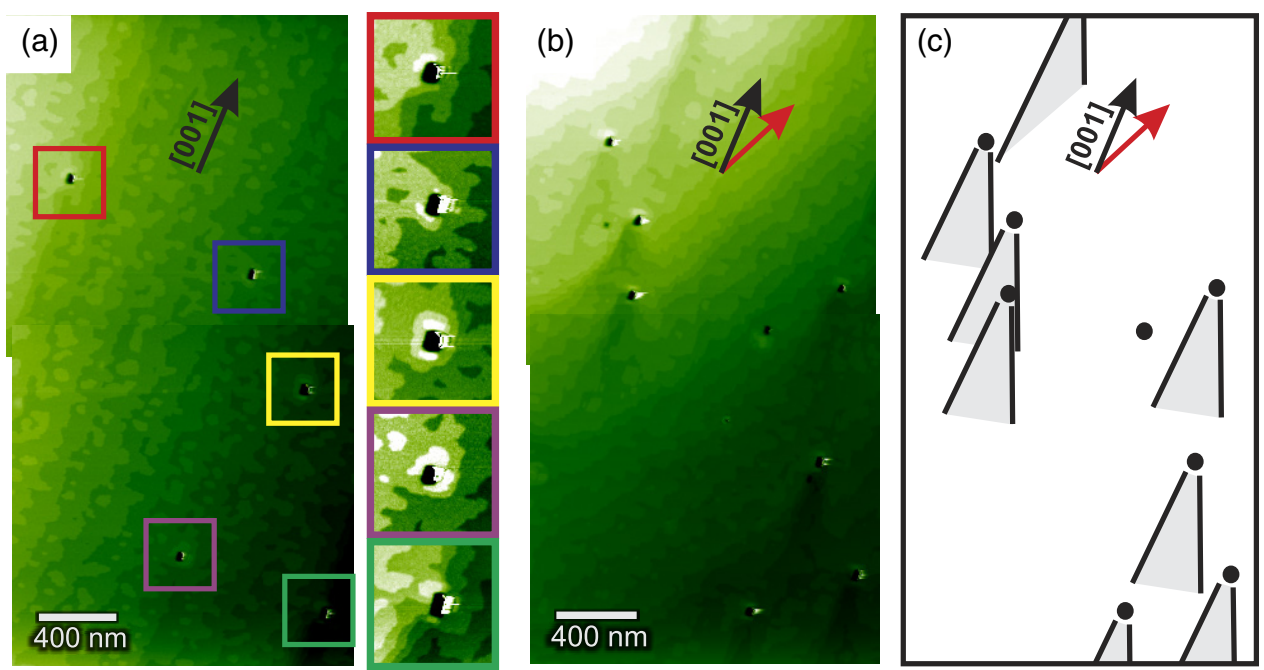

Figure 5. NC-AFM topographic images of antimony-doped $\mathrm{TiO}_{2}(110)$ obtained after annealing at $1100 \mathrm{~K}$. In total, 13 holes are visible with a mean distance of about $1 \mu \mathrm{m}$. The cut-outs extracted from (a) reveal that all holes are surrounded by small islands. This results in an appearance that resembles a volcano-like shape. The step edges can be found to run either parallel to the [001] direction (a) or enclose an angle with the [001] direction (b) as indicated by the red and black arrows. Cone-shaped troughs located at the hole sites and aligned in the [001] direction are, therefore, only observed in the case of step edges that are not parallel to [001]. The holes (black circles) and cone-shaped troughs (gray triangles) identified in (b) are sketched in (c) for illustration. 

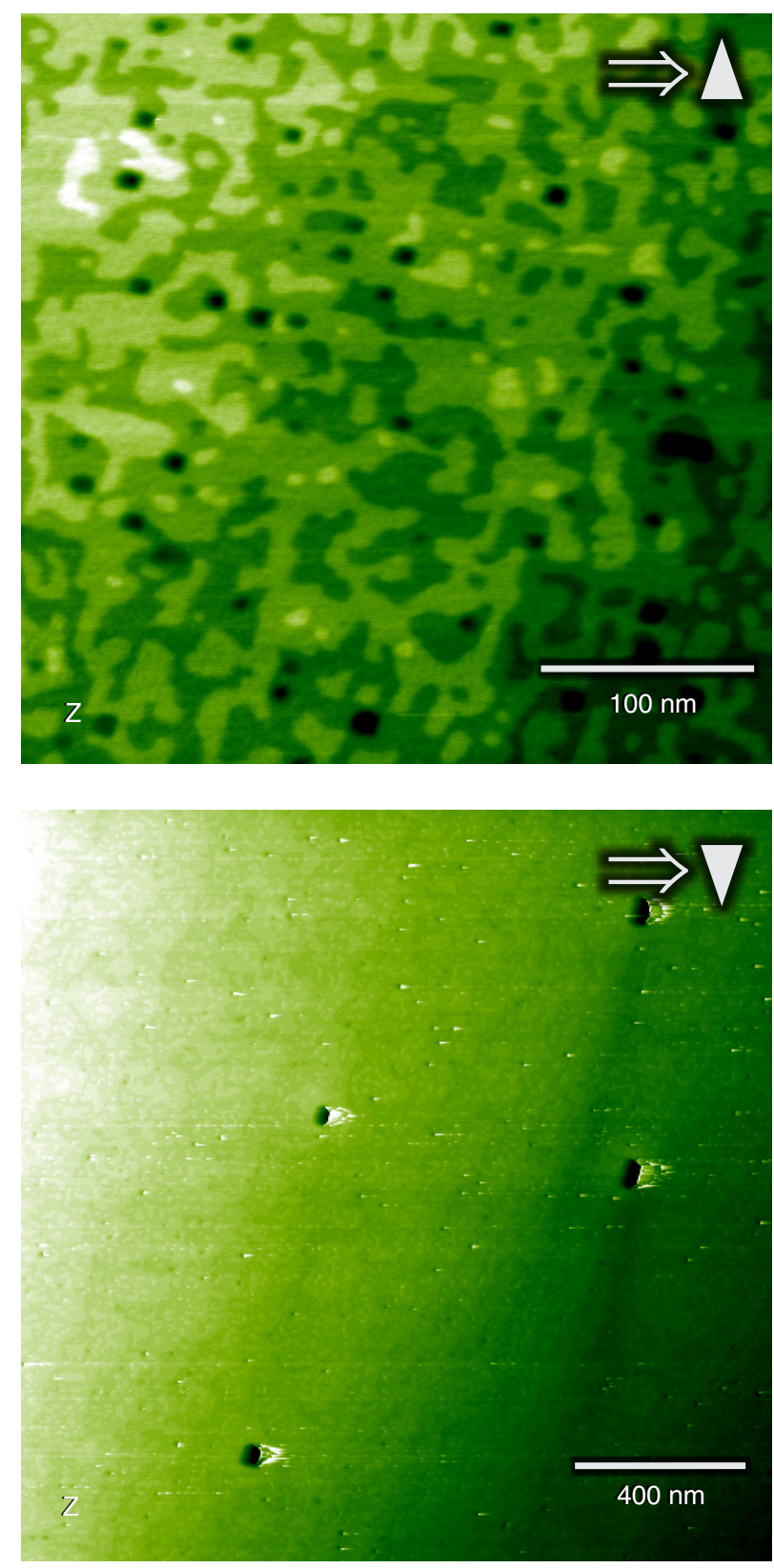

Figure 6. NC-AFM topographic images of antimony-doped $\mathrm{TiO}_{2}(110)$ obtained after annealing at $930 \mathrm{~K}$. The terraces are perforated and numerous small islands exist, similar to the pristine surface when annealed at the same temperature. Besides a few large and deep holes, which appear identical as observed after annealing at $1100 \mathrm{~K}$, a large number of small pits are present here. These pits are typically less than $2 \mathrm{~nm}$ deep and less than $10 \mathrm{~nm}$ wide.

All holes in figure 5(b) appear at the sharp end of a cone-shaped trough located at each hole. For illustration, these troughs are sketched in figure 5(c). The holes in figure 5(a) have no troughs. This difference can be explained by differently oriented step edges in the two images, as explained in the following. In figure 5 two typical images are presented, either showing step edges running parallel to the [001] direction (a) or step edges that enclose an angle with the [001] direction (b), as indicated by the red and black arrows. The cone-shaped troughs are also aligned in the [001] direction. Assuming that the terraces are growing in the [001] direction during annealing, the observed troughs might be explained by shadowing at hole sites.

\subsection{Influence of annealing temperature}

The images presented in figure 5 were obtained after annealing at $1100 \mathrm{~K}$ for $15 \mathrm{~min}$. For comparison similar images obtained after annealing at $930 \mathrm{~K}$ for $1 \mathrm{~h}$ are shown in figure 6 . The terraces appear perforated and covered by small islands similar to what we observed on pristine $\mathrm{TiO}_{2}(110)$ annealed at the same temperature. In contrast to the high temperature prepared doped surface, here a large number of small pits exist besides the large, deep holes. These small pits have a typical depth of 1-2 $\mathrm{nm}$ and an average nearest-neighbor distance of about $50 \mathrm{~nm}$.

Besides the two already described types of holes, a third type of hole is seldom observed after annealing at $1100 \mathrm{~K}$, which is typically less than $10 \mathrm{~nm}$ deep, but extends over several hundred nanometers. Two of this kind are shown in figure 7. A detailed analysis of the flat holes revealed that the typical surface structure with step edges of monatomic height can be found at the bottom of the holes, as indicated by figure 8 . Besides these flat, extended holes also deep holes with their cone-shaped trough as introduced in figures 5(b) and (c) exist in the surrounding surface region.

The images presented in figures 5(b) and 7 are obtained after the very same preparation cycle at macroscopically different surface areas. However, the size and number of holes are different. Taking the form and flatness of terraces as the reference for the annealing temperature and assuming large, island-free terraces to result from higher temperature preparation, the situation found in figure 7 might be explained by an annealing temperature slightly lower than $1100 \mathrm{~K}$. Due to different thermal contact to the silicon stripe used for resistive heating of the sample, slightly different annealing temperatures at macroscopically different sample sites are conceivable. Obviously, the number as well as the size of the holes and pits crucially depend on the exact annealing procedure. Thus, it might be possible to control the size and density of the pits by properly tuned annealing parameters.

\subsection{Summary}

In the following, we outline a possible scenario for explaining the observed structural characteristics. Antimony induces a large lattice stress in the titania crystal as already suggested by the too large ionic radius of $\mathrm{Sb}^{3+}$. Actually, an $\mathrm{Sb}^{3+}$ ion requires almost double the volume an $\mathrm{Ti}^{4+}$ ion occupies when considering the ionic radii. Although $\mathrm{Sb}^{5+}$ ions nearly perfectly fit into the crystal, they cannot remain alone as this would lead to charging of the crystal. Consequently, antimony cannot be integrated into the titania lattice at all. Thus, antimony is observed to leave the titania crystal when mobile enough. Upon vacuum annealing, antimony can segregate to the surface, forming segregation channels that appear as nanosized volcanos. At low annealing temperature, only antimony from the upper layers might have enough thermal 

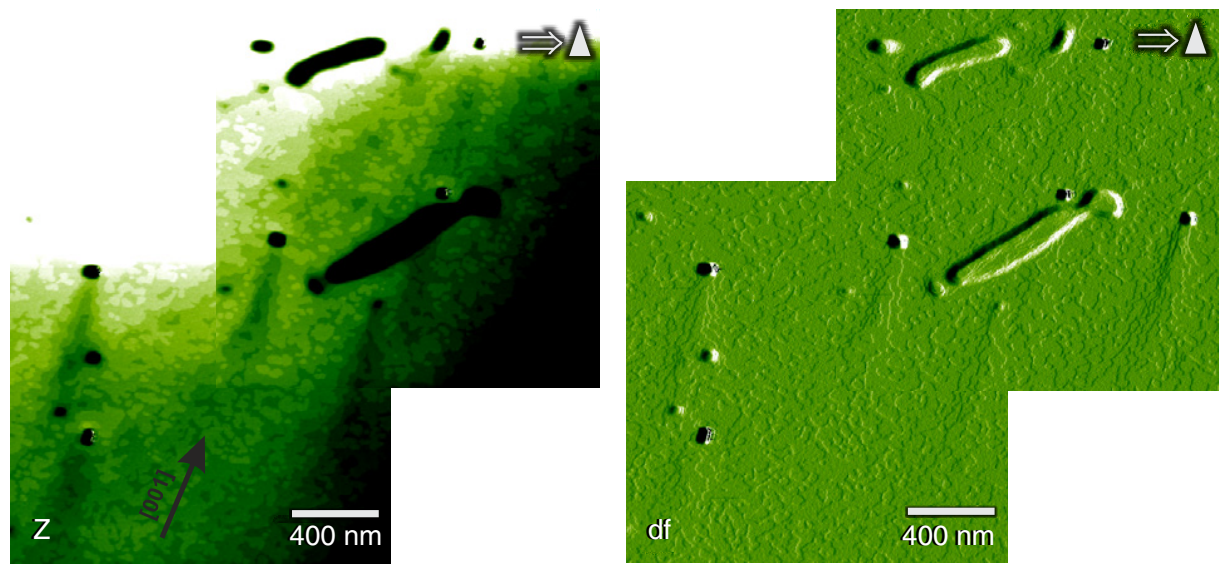

Figure 7. NC-AFM topography and detuning images of antimony-doped $\mathrm{TiO}_{2}(110)$. The images represent seldom observed flat holes that extend over several hundred nanometers. Besides, also deep holes with their cone-shaped trough as introduced in figure 5 can be observed in the surrounding area.
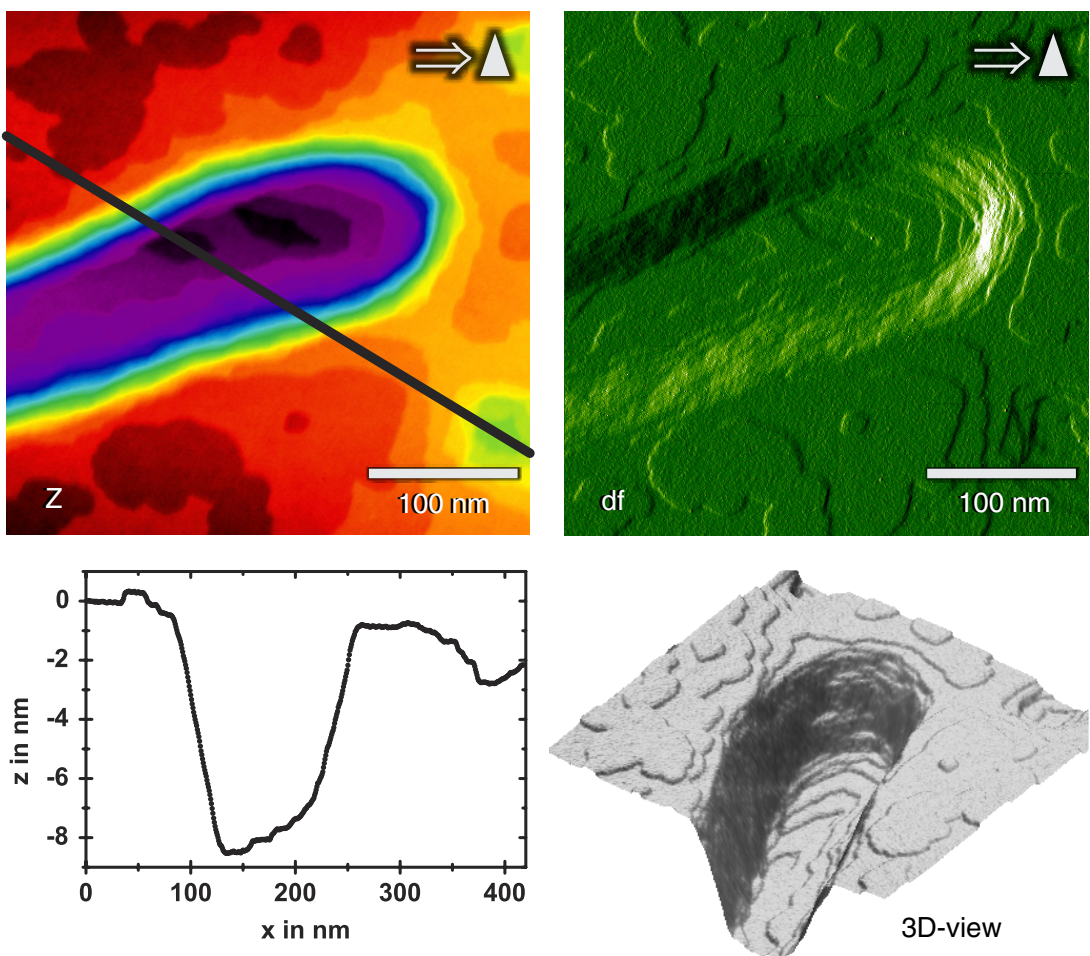

Figure 8. NC-AFM topography and detuning image of antimony-doped $\mathrm{TiO}_{2}(110)$ presenting a detail of the flat extended hole introduced by figure 7. The images as well as the three-dimensional plot of the topography image reveal that the surface at the bottom of the extended holes appears normal. From the height profile (position indicated by black line) the depth of this particular hole is measured to be about $8 \mathrm{~nm}$.

energy to reach the surface. Thus, a large number of small, flat pits is created.

At higher annealing temperature the mobility of antimony dopants is increased. Hence, material from deeper layers can also segregate to the surface. Furthermore, more material is segregated on a shorter timescale. The large and deep holes, created upon high temperature annealing, indicate that the sample surface is almost exploding as soon as antimony gets enough thermal energy to leave the bulk. In this case, instead of numerous small pits, only a few large segregation channels are established to reduce the surface energy. These holes are rather homogeneously distributed. Cone-shaped troughs are observed to exist close to hole sites, which might result from shadowing at these holes. A possible explanation is that terraces growing during annealing are distorted at the holes. The erupted material consisting of $\mathrm{TiO}_{2}$ forms new islands around the holes resulting in the volcano-like appearance. Antimony, erupted through the holes, is adsorbed as clusters on top of the surface.

\section{Conclusion}

In conclusion, the antimony-doped surface was found to exhibit terraces, steps and a row pattern similar to what is typically observed on pristine titania. However, the 
terraces were covered by a dense layer of clusters, which was tentatively explained by antimony-rich clusters.

Holes with depths ranging from a few up to more than a hundred monatomic steps were observed. The width and depth of these holes were found to crucially depend on the annealing procedure. The deepest holes were surrounded by small islands, resembling a volcano-like shape.

The different observed structures were explained by a simple model considering the ionic radii of the dopant atoms. Our findings draw a picture of weakly integrated antimony in antimony-doped titania. These results, thus, also indicate why an excess of antimony beyond the optimum ratio of 1.5 is unfavorable for photocatalytic applications, as the additional antimony ions cannot be integrated into the bulk. Moreover, the presented study revealed another interesting phenomenon. It seems to be possible to create nanosized holes in a controlled way by tuning the annealing parameters of antimony-doped $\mathrm{TiO}_{2}$ (110). This might be an appropriate strategy for creating nanopatterned surfaces with macroscopic techniques.

\section{Acknowledgments}

Financial support from the Deutsche Forschungsgemeinschaft (DFG) through an Emmy Noether grant is gratefully acknowledged. The present work was partly supported by a Grant-in-Aid for Scientific Research on Priority Areas [477] 'Molecular Science for Supra Functional Systems' from the Ministry of Education, Culture, Sports, Science and Technology, Japan.

\section{References}

[1] Diebold U 2003 Surf. Sci. Rep. 48 53-229

[2] Fujishima A, Zhang X T and Tryk D A 2008 Surf. Sci. Rep. 63 515-82

[3] Fujishima A, Rao T N and Tryk D A 2000 J. Photochem. Photobiol. C 1 1-21

[4] Fujishima A 1972 Nature 238 37-8

[5] Chen X and Mao S S 2007 Chem. Rev. 107 2891-959

[6] Choi W Y, Termin A and Hoffman M R 1994 J. Phys. Chem. 98 13669-79

[7] Di Paola A, Marcì G, Palmisano L, Schiavello M, Uosaki K, Ikeda S and Ohtani B 2002 J. Phys. Chem. B 106 637-45

[8] Ikeda T, Nomoto T, Eda K, Mizutani Y, Kato H, Kudo A and Onishi H 2008 J. Phys. Chem. C 112 1167-73

[9] Bechstein R, Kitta M, Schütte J, Kühnle A and Onishi H 2009 J. Phys. Chem. C 113 3277-80

[10] Kato H and Kudo A 2002 J. Phys. Chem. B 106 5029-34

[11] Bechstein R, Kitta M, Schütte J, Onishi H and Kühnle A 2009 in preparation

[12] Shannon R D 1976 Acta Crystallogr. A 32 751-67

[13] Binnig G, Quate C F and Gerber C 1986 Phys. Rev. Lett. 56 930-3

[14] Albrecht T R, Grütter P, Horne D and Rugar D 1991 J. Appl. Phys. $69668-73$

[15] Lauritsen J V, Foster A S, Olesen G H, Christensen M C, Kühnle A, Helveg S, Rostrup-Nielsen J R, Clausen B S, Reichling M and Besenbacher F 2006 Nanotechnology 17 3436-41

[16] Shu D J, Ge S T, Wang M and Ming N B 2008 Phys. Rev. Lett. 101116102

[17] Rahe P, Bechstein R, Schütte J, Ostendorf F and Kühnle A 2008 Phys. Rev. B 77195410 